\title{
Brain oscillations and functional connectivity during overt language production
}

\author{
Arne Ewald ${ }^{1,2 *}$, Sabrina Aristei ${ }^{3}$, Guido Nolte $^{4}$ and Rasha Abdel Rahman ${ }^{3}$ \\ ${ }^{1}$ NIRx Medizintechnik GmbH, Berlin, Germany \\ ${ }^{2}$ Machine Learning Laboratory, Berlin Institute of Technology, Berlin, Germany \\ ${ }^{3}$ Department of Psychology, Humboldt-Universität zu Berlin, Berlin, Germany \\ ${ }^{4}$ Department of Neurophysiology and Pathophysiology, University Medical Center Hamburg-Eppendorf, Hamburg, Germany
}

Edited by:

Lucia Melloni, Max Planck Institute for Brain Research, Germany

\section{Reviewed by:}

Olaf Hauk, MRC Cognition and Brain Sciences Unit, UK

Michael Wibral, Goethe University, Germany

Christian A. Kell, Goethe University, Germany

\section{*Correspondence:}

Arne Ewald, NIRx Medizintechnik

$\mathrm{GmbH}$, Baumbachstr. 17, 13189

Berlin, Germany.

e-mail:mail@aewald.net
In the present study we investigate the communication of different large scale brain sites during an overt language production task with state of the art methods for the estimation of EEG functional connectivity. Participants performed a semantic blocking task in which objects were named in semantically homogeneous blocks of trials consisting of members of a semantic category (e.g., all objects are tools) or in heterogeneous blocks, consisting of unrelated objects. The classic pattern of slower naming times in the homogeneous relative to heterogeneous blocks is assumed to reflect the duration of lexical selection. For the collected data in the homogeneous and heterogeneous conditions the imaginary part of coherency $(\mathrm{ImC})$ was evaluated at different frequencies. The $\mathrm{ImC}$ is a measure for detecting the coupling of different brain sites acting on sensor level. Most importantly, the ImC is robust to the artifact of volume conduction. We analyzed the $\mathrm{ImC}$ at all pairs of 56 EEG channels across all frequencies. Contrasting the two experimental conditions we found pronounced differences in the theta band at $7 \mathrm{~Hz}$ and estimated the most dominant underlying brain sources via a minimum norm inverse solution based on the $\mathrm{ImC}$. As a result of the source localization, we observed connectivity between occipito-temporal and frontal areas, which are well-known to play a major role in lexical-semantic language processes. Our findings demonstrate the feasibility of investigating interactive brain activity during overt language production.

Keywords: overt language production, semantic interference, brain oscillations, functional connectivity

\section{INTRODUCTION}

Electroencephalographic measures have long played only a minor role in the investigation of language production. A major reason for avoiding the recording of the electroencephalogram (EEG) during overt speech was the suspicion that articulation-related artifacts such as facial muscle activity, lip and eye movements, glossokinetic potentials, and head movements may severely disrupt the signal early on (e.g., Brooker and Donald, 1980; Wohlert, 1993; Grözinger et al., 1975). To avoid articulation-induced artifacts, some EEG studies of language production employed paradigms in which overt speech was either delayed (e.g., Jescheniak et al., 2002) or replaced by silent naming (e.g., Eulitz et al., 2000) or manual responses (e.g., van Turennout et al., 1997, 1998; Schmitt et al., 2000, 2001; Abdel Rahman and Sommer, 2003; Abdel Rahman et al., 2003). One of the disadvantages of such approaches, however, is the difficulty of directly relating behavioral measures and electrophysiological correlates. Furthermore, it has been argued that meta-linguistic button-press tasks may not include all processes involved in natural language production (but, see Abdel Rahman and Aristei, 2010).

Recently, several studies have demonstrated the feasibility of combining EEG measures with overt articulation (for reviews, see Ganushchak et al., 2011; Indefrey, 2011; Strijkers and Costa, 2011). These studies have provided valuable information about the precise temporal unfolding of different processes involved in language production. For instance, semantic context effects in classic speech production paradigms such as the picture-wordinterference (PWI) paradigm (Hirschfeld et al., 2008), the semantic blocking paradigm (Aristei et al., 2011, see also Maess et al., 2002 for evidence from magnetoencephalography) or the cumulative semantic interference paradigm (Costa et al., 2009) have been shown to elicit modulations in the event-related brain potential (ERP) within a latency range of well-below $300 \mathrm{~ms}$ post-stimulus onset. These semantic context effects are assumed to reflect the selection of an appropriate lexical candidate for a semantic concept (pre-verbal message) to be expressed. For instance, in the blocking paradigm participants name objects that are presented in blocks of trials that consist of a homogeneous group of category members (e.g., all objects belong to the category of animals; homogeneous blocks) or blocks that consist of semantically unrelated objects (heterogeneous blocks). When the pictures are repeatedly named (but typically not when they are named for the first time), a semantic interference effect is observed that reflects slower naming times in the homogeneous relative to the heterogeneous condition. Aristei et al. (2011) reported ERPs elicited by semantic contexts around $200 \mathrm{~ms}$ after stimulus onset at occipito-temporal and frontal scalp regions (see also Costa et al., 2009 for similar findings). 
To summarize, a growing number of studies has revealed the feasibility of combining EEG measurements with overt speech production, yielding fine-grained temporal information on various aspects of speech production, such as bilingualism (Christoffels et al., 2007), lexical-semantic access (e.g., Hirschfeld et al., 2008; Costa et al., 2009; Strijkers et al., 2010; Aristei et al., 2011), and error monitoring (Ganushchak and Schiller, 2008, 2009). However, in contrast to this recent development concerning electrophysiological evidence on the temporal unfolding of language production, the identification of the functional networks and correlated brain dynamics associated with electrophysiological brain activity during overt speech are largely unknown. The aim of the present study was to investigate lexicalization with electrophysiological measures of functional connectivity and oscillatory brain dynamics, as described below.

Another aspect of the EEG is that its high temporal resolution in the millisecond range provides a means to capture and investigate rhythms generated by large neuronal populations in different compartments inside the brain at different points in time. Furthermore, functional synchronization of these oscillations is assumed to play a key role as a communication mechanism in the brain (Singer, 1999; Engel et al., 2001; Varela et al., 2001; Fries, 2005). Outside the area of language production the investigation of the interplay between neuronal populations has, hence, become a growing field of research in order to gain a deeper understanding of functional information processing within the brain in different experimentally controlled tasks.

The purpose of the present study was twofold. First, we wanted to identify the brain dynamics associated with the functional networks involved in semantic-lexical access during language production. To this end, we analyzed brain connectivity measures elicited during overt object naming in a semantic blocking paradigm, as detailed below. Second, we aimed to demonstrate the feasibility and the procedure of applying reliable connectivity measures.

In contrast to the good temporal resolution, EEG measurements suffer from a poor spatial resolution. As brain activity is measured non-invasively on the scalp, the electric fields generated by neuronal sources propagate through the head and even a single source can be recorded by many of the EEG electrodes depending on the location and orientation of the source (e.g., Nunez et al., 1999). Therefore, in presence of many macroscopically measurable brains sources, the EEG captures a mixture of these sources in each channel. This effect, most often termed volume conduction or field spread, is known to be crucial especially in the context of analysis of brain connectivity. A functional relationship between the signals measured at two EEG sensors can generally not be interpreted as a relationship between underlying sources due to the mixing of sources into sensors. One way to tackle this problem is to first calculate the time series of the brain sources and then apply an appropriate connectivity measure. Unfortunately, the solution of the so called inverse problem, i.e., the calculation of source activity from EEG measurements, is not uniquely solvable, and hence, every estimate is error prone (Baillet et al., 2001). Furthermore, volume conduction can play an important role within the estimation procedure of connectivity on source level. Even the application of quite robust and widely used inverse methods (e.g., beamformers) can lead to substantial misinterpretation of the results (Schoeffelen and Gross, 2009).

To overcome the introduced limitations we apply a different approach for the calculation of functional connectivity. Within the present study we use a connectivity measure on sensor level with a special treatment for volume conduction, namely the imaginary part of coherency (ImC) introduced by Nolte et al. (2004). We shortly recover the basic concept and the special role of the imaginary part. For a more detailed review and a discussion of the relationship to other methods such as phase-locking (Lachaux et al., 1999), please consider the original research paper. The key feature of the ImC is its "robustness to the artifact of volume conduction" indicating that a significant deviation from zero of the applied measure cannot be due to independent sources that are mapped simultaneously in different EEG channels. As the interpretation of sensor level connectivity in terms of brain sources is not unique, we also apply a weighted minimum norm inverse solution on the most dominant subspace of the imaginary part of the cross-spectrum and demix the corresponding brain sources with the assumption of spatially distributed sources (Marzetti et al., 2008). Hence, we obtain a rough but reliable picture of interacting brain sources.

Evidence of language related brain oscillatory activity, even though lacking for language production, is available for different sub-processes of language comprehension. Previous findings suggest the involvement of two frequency bands in lexical-semantic operations, the theta $(4-7 \mathrm{~Hz})$ and alpha $(8-12 \mathrm{~Hz})$ band. Theta oscillations have been shown to reflect processes involved in lexical-semantic retrieval. For instance, theta event-related synchronization (ERS) responses increase for words relative to pseudowords in lexical decision tasks (Krause et al., 2006). Similarly, during lexical decision tasks noun semantic properties, that is, nouns with either visual or auditory properties (e.g., colors or sounds) affect theta oscillatory activity at different scalp regions corresponding to brain areas typically associated with visual or auditory processing (e.g., Bastiaansen et al., 2008). Furthermore, in reading tasks, left-lateralized temporal theta activity is modulated by lexical factors such as word category class (e.g., open vs. close class words; Bastiaansen et al., 2005). Finally, oscillatory theta activity changes have been reported in association with syntactic number violations, grammatical gender violations, and semantic violations in reading, suggesting an association with lexical information processing (e.g., Bastiaansen et al., 2002; Hagoort et al., 2004). In addition to oscillation changes in theta frequencies, power decrease in alpha frequency band has been associated with enhanced semantic processing (e.g., Klimesch et al., 1997; Rohm et al., 2001; for a review, see Klimesch, 1999). Together, these findings suggest that brain oscillations in the alpha and theta band reflect the retrieval of lexical-semantic information in language comprehension (e.g., Bastiaansen et al., 2005; for the role of oscillations at low frequency bands in language processing, see also Arnal et al., 2011). Because lexical and semantic representations and/or operations may be shared at least to some extent by the language production and perception system (e.g., Indefrey and Levelt, 2004), we expected similar effects in the alpha and theta frequency range at temporal and frontal regions to reflect the functional networks involved in semantic-lexical retrieval during 
language production. We tested these assumptions with a semantic blocking paradigm in which pictures of objects are named in categorically homogeneous or heterogeneous blocks of trials (e.g., Kroll and Stewart, 1994; Damian et al., 2001; Belke et al., 2005), assuming that this task taps into lexical-semantic processing stages during language production.

\section{MATERIALS AND METHODS PARTICIPANTS}

Seventeen females and seven males, aged 20-34 years $(N=24)$, were paid for their participation in the experiment or received partial fulfillment of a curriculum requirement. All participants were native German speakers and reported normal or corrected-to-normal visual accuracy and normal color vision.

\section{MATERIALS}

The picture set consisted of 125 color photographs of common objects from 25 semantic categories. All pictures were presented in semantically homogeneous blocks (all objects were category members, e.g., insects, beverages, kitchen utensils, etc.) or in heterogeneous blocks consisting of semantically unrelated objects. The size of the photographs was $3.5 \mathrm{~cm} \times 3.5 \mathrm{~cm}$ at an approximate viewing distance of $90 \mathrm{~cm}$ from the monitor. All picture names span a broad range of lexical frequency (normalized lemma frequency: from 0.016 to 102.68 ; as per DLex database) and word length (from 2 to 13 letters). Because all pictures (and thus, all names) appeared equally often in all conditions, stimulus characteristics can be excluded as a source for experimental effects and matching procedures are thus not necessary.

\section{PROCEDURE}

Prior to the experiment, participants were familiarized with all objects and their names as follows: first, all photographs were presented in random order on the screen, and participants were asked to name each object. If necessary, they were corrected or the picture name was provided by the experimenter. Then participants were given a printed color sheet with all objects and their names printed below.

Stimulus presentation and response recording was controlled by Presentation software (Neurobehavioral Systems). Each trial began with a fixation cross in the center of a light gray screen. After $500 \mathrm{~ms}$, a picture was presented until vocal response, with a maximum duration of $2 \mathrm{~s}$. Vocal responses were recorded with a microphone and naming latencies were measured with a voice-key. The voice-key, connected to the microphone, was triggered when the sound pressure reached a predefined level, indicating the onset of the naming response. Naming accuracy and voice-key functioning were monitored online by the experimenter. All pictures were presented five times in semantically homogeneous blocks that consisted of sub- or superordinate-level category coordinates (e.g., insects or animals, respectively), and heterogeneous blocks. Throughout the manuscript, we focus on the strongest comparison between subordinate-level homogeneous blocks consisting of closely related objects and heterogeneous blocks of unrelated objects. Participants were instructed to name each object as fast and accurately as possible.

The order of semantic blocking conditions and the order of the individual categories was counterbalanced across participants.
The session was subdivided by short breaks during which participants could rest and execute eye and small body movements. The whole session with 1875 trials lasted about $95 \mathrm{~min}$.

\section{EEG RECORDING AND ANALYSIS}

The continuous electroencephalogram (EEG) was recorded with sintered $\mathrm{Ag} / \mathrm{AgCl}$ electrodes from 56 sites according to the extended 10-20 system, referenced to the left mastoid, and at a sampling rate of $500 \mathrm{~Hz}$ (bandpass $0.032-70 \mathrm{~Hz}$ ). The horizontal and vertical electrooculogram was measured with external electrodes attached to the left and right canthi of both eyes and beneath and above the left eye. Electrode impedance was kept below $5 \mathrm{k} \Omega$ for all scalp electrodes and below $10 \mathrm{k} \Omega$ for peripheral sites.

For the analysis, the data were down sampled to $250 \mathrm{~Hz}$ and filtered with notch filter at $50 \mathrm{~Hz}$ for the removal of electrical component noise. Additionally, the data were re-referenced to linked mastoids. All 56 head electrodes were used for the spectral and the connectivity analysis. According to the experimental setup, the data were split into epochs belonging to the two blocking conditions (homogeneous and heterogeneous; a detailed description of the parameters can be found in section Electrophysiological Data), and certain epochs were not taken into account due to a variance based artifact rejection procedure. For the artifact removal, first the median of the maximal absolute value for all epochs and channels is calculated. Then any epoch in which at least one channel has a maximal absolute value above threshold, set to three times the median, is removed. The automated artifact removal procedure excluded 17.15 and $17.51 \%$ epochs for the homogenous and heterogeneous condition respectively (analyzed epochs: 9115 for homogeneous and 9088 for heterogeneous blocks). Furthermore, because semantic interference develops across repetitions and is absent for the first stimulus presentation (e.g., Damian et al., 2001), epochs belonging to the first stimulus presentation in each condition were excluded (see also Behavioral Data). Hence, we focused our analyses on the effects emerging during the last four repetitions (from two to five). Finally, the analysis of the data in terms of connectivity was performed as a grand average over all participants.

As an initial view on the data we inspected the power in each EEG channel. Based on the analysis of power in dependence on frequency and time we determined parameters needed for further proceeding, such as the relevant time interval for the calculation of coherency (see Electrophysiological Data). The power over time and frequency was calculated based on the cross-spectrum in several time intervals. The cross-spectrum between channels $i$ and $j$ is defined as

$S_{i j}(f)=\left\langle x_{i}(f) x_{j}^{*}(f)\right\rangle$

where $x^{i}(f)$ denotes the complex Fourier transform in a certain time interval, ${ }^{*}$ the complex conjugate ${ }^{1}$ and $\langle\cdot\rangle$ denotes the expectation value that is usually approximated by averaging over a sufficiently large number of trial (Bendat and Piersol, 1971). If

\footnotetext{
${ }^{1}$ As the FFT is complex valued we obtain $x_{i}(f)=a+i b$. The complex conjugate is defined by inverting the imaginary part: $x_{i}^{*}(f)=a-i b$.
} 
we set $i=j$, we obtain the auto spectrum $S_{i j}(f)$ and, hence, the power of channel $i$.

For the inspection of the individual channel power over frequency and time we constructed epochs around each stimulus lasting from $1 \mathrm{~s}$ before to $2 \mathrm{~s}$ after each picture presentation. These epochs were divided into segments of $1 \mathrm{~s}$ length and the center of each segment was shifted by $100 \mathrm{~ms}$. Within these segments a Hanning windowed fast Fourier transform (FFT) was computed. By averaging the absolute square over all epochs we obtain a spectrogram for each channel as shown in Figure 2. The grand average over all participants for the calculation of the spectrogram and for the ImC was carried out by averaging the individual crossspectra for each subject at each frequency and in each experimental condition.

As stated shortly in the introduction we applied a coherency based approach with a special treatment for volume conduction to detect true functional connectivity on sensor level. The complex quantity coherency ${ }^{2}$ is defined as the normalized cross-spectrum:

$C_{i j}(f)=\frac{S_{i j}(f)}{\sqrt{S_{i i}(f) S_{j j}(f)}}$.

Essentially, coherency is a measure of the consistency of a phase relationship between two signals with respect to distinct stimuli. In many EEG applications coherence is shown as the absolute value of coherency. But as a single brain source is mapped into many channels, a consistent phase relationship between the channel signals can arise without truly interacting brain sources. To overcome this problem and to diminish the effect of these spurious interactions, Nolte et al. (2004) proposed to focus on the ImC. We shortly recover the basic concept and the special role of the imaginary part. For a more detailed review and for the relationship to other methods such as phase-locking (Lachaux et al., 1999), please consider the original research paper.

The linear mixture of sources to EEG channels is mathematically expressed in the frequency domain as

$$
x_{i}(f)=\sum_{k=1}^{K} a_{i k} s_{k}(f),
$$

with $x_{i}(f)$ being the FFT of the measured signal in channel $i$ in which $K$ sources $s_{k}(f)$ are linearly mapped. Assuming an instantaneous mapping, the mixing coefficients $a_{i k}$ are real valued as the phases of the source signal are not distorted. If we further assume independent, i.e., non-interacting sources $s_{k}(f)$ and insert Eq. 3 into Eq. 1 we obtain for the cross-spectrum

$$
\begin{aligned}
S_{i j}(f) & =\left\langle x_{i}(f) x_{j}^{*}(f)\right\rangle=\sum_{k k^{\prime}} a_{i k} a_{j k^{\prime}}\left\langle s_{k}(f) s_{k^{\prime}}^{*}(f)\right\rangle \\
& =\sum_{k} a_{i k} a_{j k}\left\langle s_{k}(f) s_{k}^{*}(f)\right\rangle=\sum_{k} a_{i k} a_{j k}\left\langle\left|s_{k}(f)\right|^{2}\right\rangle
\end{aligned}
$$

\footnotetext{
${ }^{2}$ The terminology alters in different publications. With coherence most often the absolute value of coherency is meant. To distinguish these two quantities clearly, we refer to the complex quantity as coherency.
}

and we observe that the quantities $S_{i j}(f)$ are real valued. As the normalization for the computation of coherency, as shown in Eq. 2 , is also real valued, coherency is real valued for independent sources. Note that this holds for an arbitrary number of sources and regardless of whether the source dynamic is linear or nonlinear. The assumption of an instantaneous mapping holds for the frequency of interests in EEG, as the quasi-static approximation of the Maxwell equations appears to be valid up to $2 \mathrm{kHz}$ (Plonsey and Heppner, 1967; Stinstra and Peters, 1998).

To test the results of the analysis in terms of significance and correct for multiple comparison we applied the false discovery rate (FDR; Benjamini and Hochberg, 1995; Storey, 2002). In our case of bivariate interactions on sensor level, the FDR controls for the rate of false detections of interacting pairs. Analyzing the outcome of coherency based measures of functional connectivity, the FDR, with a certain $q$-level given, states that $(1-q) \times 100 \%$ of the observations (interacting pairs) can be expected to be true observations. The FDR has been successfully applied in neuroimaging (Genovese et al., 2002) and is, generally spoken, less conservative than other correction procedures such as the Bonferroni method.

As connectivity patterns on sensor level do not necessarily provide interpretable information about the locations of the sources inside the brain, we, furthermore, applied inverse calculations. These are based on the imaginary part of the cross-spectrum as a reliable measure for neuronal interactionsrobust to volume conduction. Please note that we do not claim to provide a full and exact picture of source activity but focus on the main interactions differing in the two experimental conditions. The aim is to yield a qualitative estimate of which sources give rise to the previously determined connectivity pattern on sensor level. To focus on the most prominent interaction at a given frequency, we performed a subspace decomposition of the imaginary part of the cross-spectrum via a singular value decomposition (SVD). This leads to

$\operatorname{Im}(S(f))=U(f) D(f) V^{T}(f)$

where $U$ and $V$ are orthonormal matrices and $D$ is a diagonal matrix with positive elements in the diagonal. The eigenvectors $u_{1}$ and $u_{2}$ belonging to the largest singular values $s_{\max , 1}$ and $s_{\max , 2}$ can be seen as the topographies of two sources describing the major interaction at a particular frequency $f$. Here, they serve as an input for a weighted minimum norm estimate (WMNE) inverse solution (Hauk, 2004) to calculate the sources giving rise to the topographies $u_{1}$ and $u_{2}$. In addition to the pure inverse calculation, the sources have to be demixed. The problem arises as we deal with interactions and antisymmetric cross-spectral matrices, Hence, the singular values are degenerate (see Nolte et al., 2006; Ewald et al., 2011). In particular, this means that the two eigenvectors $u_{1}$ and $u_{2}$ belong to one singular value $s_{\max , 1}=s_{\max , 2}$ and each linear combination of the two eigenvectors is again an eigenvector. In other words, $u_{1}$ and $u_{2}$ are not unique and have to be determined using additional assumptions. In order to demix the estimated brain sources we apply Minimum Overlap Component Analysis (MOCA) which is based on the assumption of spatially separated sources (Marzetti et al., 2008). 


\section{RESULTS \\ BEHAVIORAL DATA}

Mean response times (RTs) for correct trials, standard errors of means, and mean percentages of errors in the experimental conditions are presented in Table 1. Trials with incorrect naming, disfluencies, mouth clicks, or vocal hesitations and trials with voice-key failures or malfunctioning were discarded from the RT analysis. Trials with naming latencies that deviated from the participant's mean by more than 3 standard deviations were also excluded from the analysis.

Analyses of variance (ANOVAs) on RTs were performed with the within-participants factors semantic blocking (semantically homogeneous, heterogeneous), and repetition. All ANOVAs were calculated with participants and items as random factors $\left(F_{1}\right.$ and $F_{2}$, respectively). Where necessary, the reported $p$-values are corrected for the degrees of freedom using the Huynh-Feldt procedure (Huynh and Feldt, 1976).

Analyses of variance yielded significant main effects of semantic blocking, $F_{1}(1,23)=11.2, \mathrm{MSE}=2755, p=0.003 ; F_{2}(1$, $124)=55.4, \mathrm{MSE}=3004, p<0.001$, repetition, $F_{1}(4,92)=223.6$, $\mathrm{MSE}=335, p<0.001 ; F_{2}(4,496)=304, \mathrm{MSE}=1380, p<0.001$, and an interaction between semantic blocking and repetition, $F_{1}(4,92)=35.5, \mathrm{MSE}=390, p<0.001 ; F_{2}(4,92)=82.5$, $\mathrm{MSE}=1020, p<0.001$ that reflects the observation that semantic blocking effects are not present in the first naming trials. Because it has repeatedly been shown in the blocking paradigm that interference is typically not observed for the first picture presentation (see for instance Damian et al., 2001; Belke et al., 2005; Abdel Rahman and Melinger, 2007), we conducted an additional analysis on the data excluding the first naming trials. Furthermore, because interference effects typically stabilize after the first presentation, and because we are mainly interested in the basic effects of semantic interference in brain oscillations, we confined all further behavioral and electrophysiological analyses to the collapsed presentations two to five. As expected, these analyses revealed a highly significant main effect of semantic blocking in RTs, $t_{1}(23)=3.7$, $p=0.001 ; t_{2}(124)=7.9, p<0.001$.

\section{ELECTROPHYSIOLOGICAL DATA}

As described in the introduction we conducted EEG analyses in terms of functional connectivity on the sensor level. In a first step we investigated the power in each channel as a function of frequency and time in the two blocking conditions (homogeneous

Table 1 | Mean response times, standard errors of means, and mean error rates in semantically homogeneous and heterogeneous naming conditions.

\begin{tabular}{|c|c|c|c|c|c|c|}
\hline \multirow[t]{2}{*}{ Presentation } & \multicolumn{2}{|c|}{ Mean RT (ms) } & \multicolumn{2}{|c|}{ SE } & \multicolumn{2}{|c|}{ ER (\%) } \\
\hline & Hom. & Het. & Hom. & Het. & Hom. & Het. \\
\hline 1 & 738 & 777 & 12 & 14 & 7.8 & 9.7 \\
\hline 2 & 685 & 656 & 11 & 12 & 4.2 & 3.8 \\
\hline 3 & 681 & 650 & 12 & 11 & 4.3 & 3.5 \\
\hline 4 & 694 & 648 & 13 & 12 & 3.8 & 3.7 \\
\hline 5 & 691 & 645 & 12 & 11 & 3.3 & 3.4 \\
\hline
\end{tabular}

and heterogeneous). A grand average of the power spectrum over all participants for each individual EEG channel is shown in Figure 2 in the upper part for both conditions. The lower part of Figure 2 exemplarily magnifies the power spectrum over time for channel "Pz." The choice of "Pz" is arbitrary and for illustration purposes only and representative for the temporal evolution of different rhythms observed at many EEG channels.

Visual inspection of the spectrograms reveals changes in several frequency bands. Rhythms in the gamma (broad band around $35 \mathrm{~Hz}$ ) and theta band (around 3-7 Hz) develop in the 1-s time interval after stimulus onset. In the same time interval a desynchronization of the alpha rhythm $(8-12 \mathrm{~Hz})$ is observed. In a slightly later interval (about $1000 \mathrm{~ms}$ after stimulus onset) weak oscillation changes in the beta range (around $17 \mathrm{~Hz}$ ) can be also detected.

Please note that we do not aim to provide a profound or exhaustive interpretation of all ongoing oscillatory activity during the presented experiment. The main purpose of this paper is the investigation of reliable effects of functional connectivity and, therefore, no statistical analysis is performed for the presented spectrograms. Hence, the reason for inspecting the spectrograms is the coarse investigation of the temporal evolution of oscillations in order to determine a proper time interval $t$ for the calculation of the ImC. Previous ERP studies of semantic blocking effects (e.g., Maess et al., 2002; Aristei et al., 2011) revealed that the time interval of interest includes approximately the first $500 \mathrm{~ms}$ post-stimulus (see Figure 1). However, because oscillations need more time to develop a stable state, we chose a larger time interval of $t=(0 \ldots 1 \mathrm{~s})$ for the epochs (see EEG Recording and Analysis) which are tested for phase consistency over trials and participants.

After having determined an appropriate time interval we inspected the ImC as a measure for functional connectivity on sensor level, robust to the artifact of volume conduction. Figure 3

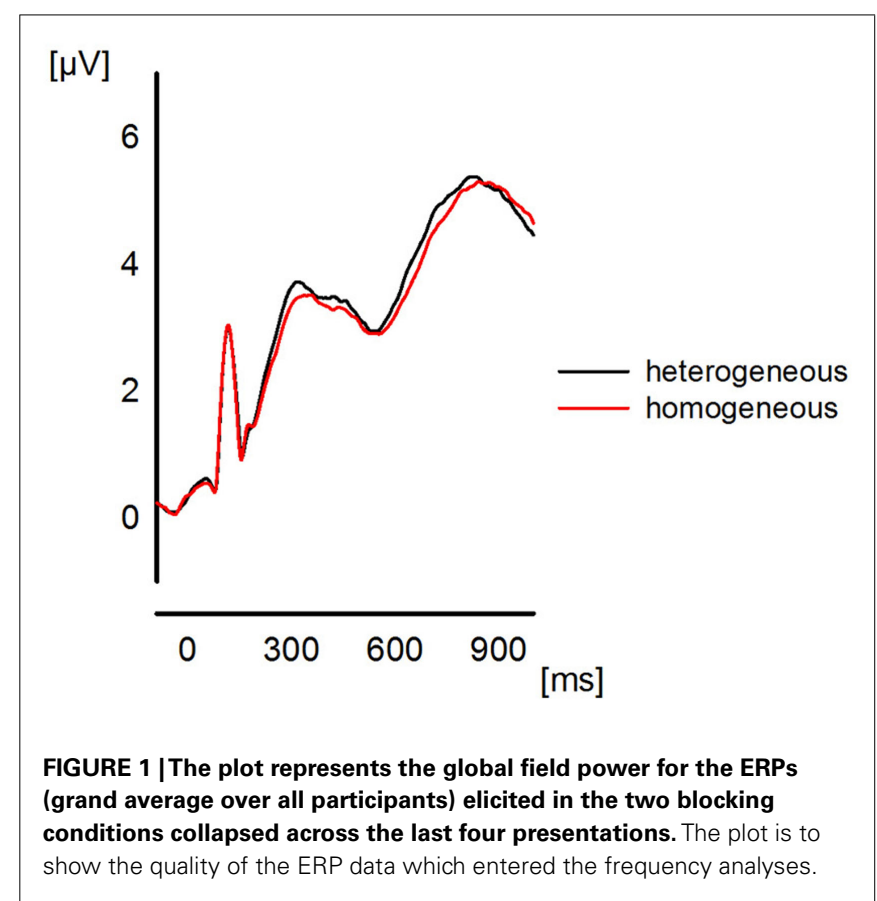




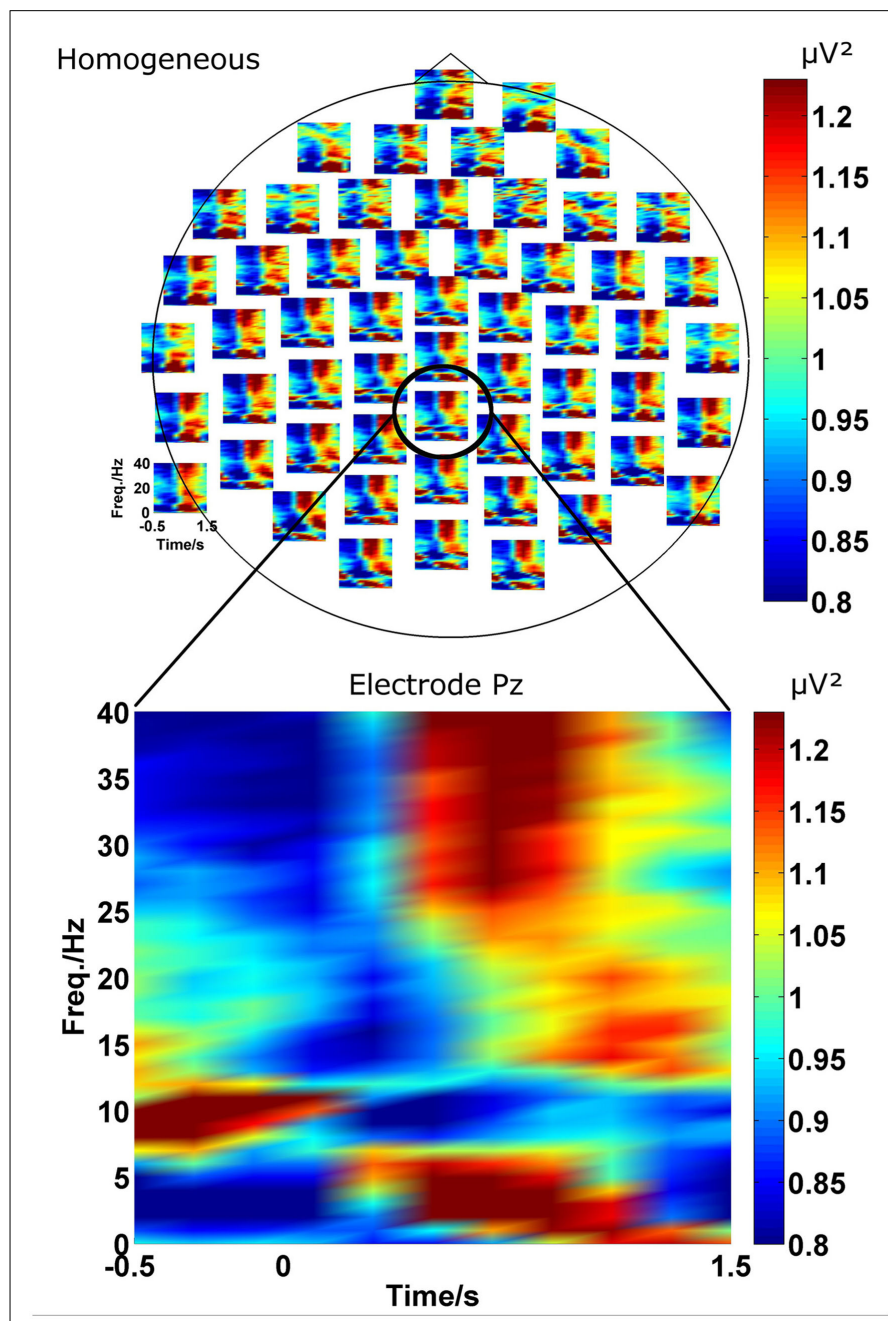

FIGURE 2 |The two plots on the top represent the spectrogram for the homogeneous (left) and heterogeneous (right) blocking condition. For a more detailed view, the spectrogram at Pz (bottom) is exemplarily magnified.

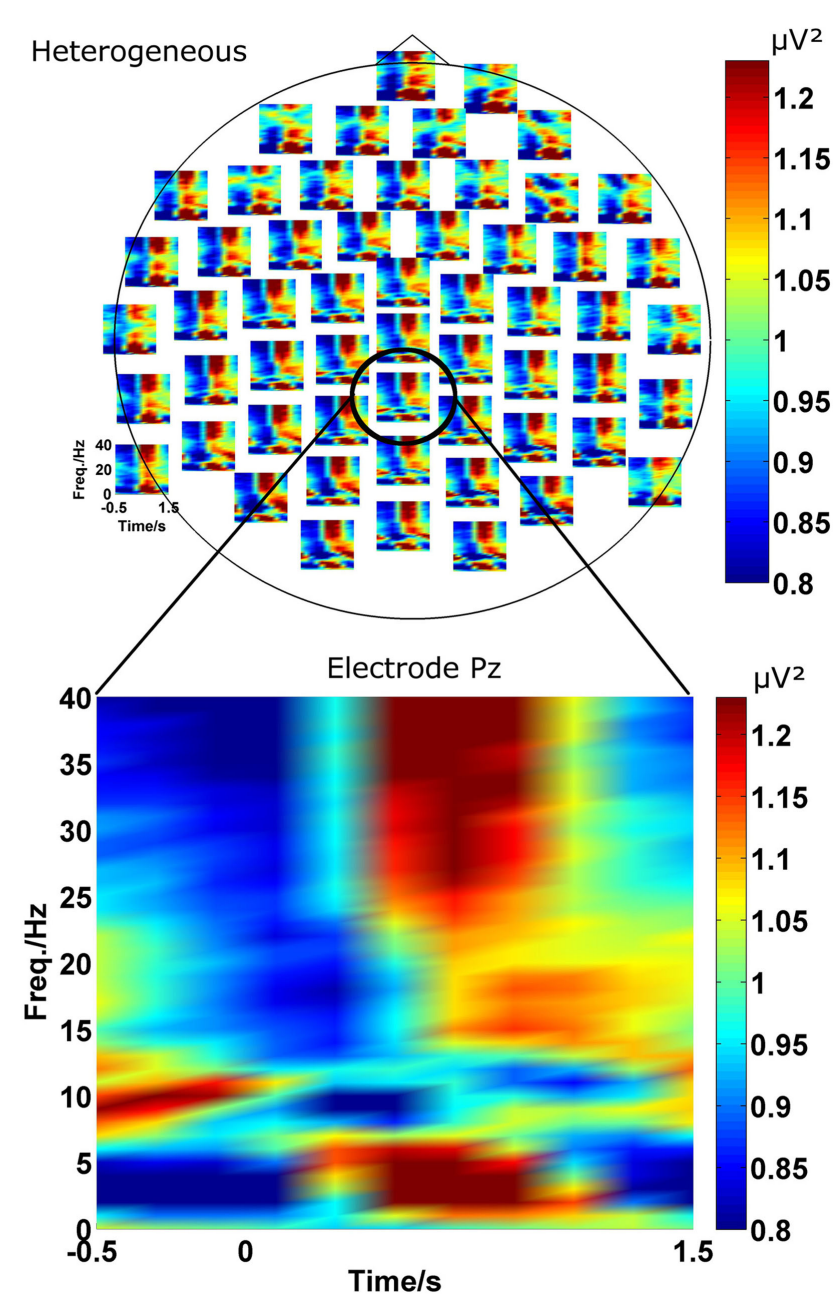

In both conditions ongoing oscillations in theta, alpha, beta, and gamma frequency bands evolving after stimulus onset can be observed. Furthermore, the spectrograms are similar in both conditions. shows the ImC for each channel pair over frequency separately for the two blocking conditions as well as for their difference.

The examination of power spectra over time reveals remarkable similarities between the two conditions (see Figure 2). Similarly, in the ImC values the two conditions also share a common pattern, with a pronounced peak in the alpha $(10 \mathrm{~Hz})$ and theta (around 4-5 Hz) bands. Although having applied an artifact correction procedure, we take a conservative view point and consider activity in the lowest frequency band (about $1-2 \mathrm{~Hz}$ ) as a not trustworthy interaction as artifacts related to eye movements occur in this frequency range (e.g., Woestenburg et al., 1983). Furthermore, activity in a very broad gamma band between only a few electrode pairs are likely to correlate with muscle activity (e.g., Brunner et al., 1996) generated during articulation. Nevertheless, this effect vanishes with respect to the difference of both conditions.

In contrast to the rather similar ImC in both conditions, a different scenario emerges from the inspection of the ImC difference between the two blocking conditions (Figure 3 right). A peak at
$7 \mathrm{~Hz}$ can be observed in the single conditions (Figure 3 left) and it becomes evident after averaging over the absolute value of all individual channel differences (Figure 3 bottom right). This pattern suggests that in terms of connectivity the most prominent difference between homogeneous and heterogeneous blocking conditions occurs at a frequency of $7 \mathrm{~Hz}$.

In order to validate this effect statistically and to test for significance, we applied a permutation test (Moore and McCabe, 2003). In $N=2000$ runs, the epochs were randomly assigned to one of the two experimental conditions. In a second step, we recalculated on these permuted data the average over all channel pairs of the absolute value of individual channel differences in ImC. The results are illustrated in Figure 4. The upper plot shows the results of the permutation test as a box plot for all frequencies up to $30 \mathrm{~Hz}$. The bar inside the box indicates the median of all permutations and the outer borders of the box the respective quartiles. The whiskers show all permutation results outside of the quartile range. Furthermore, the originally measured values are overlaid 

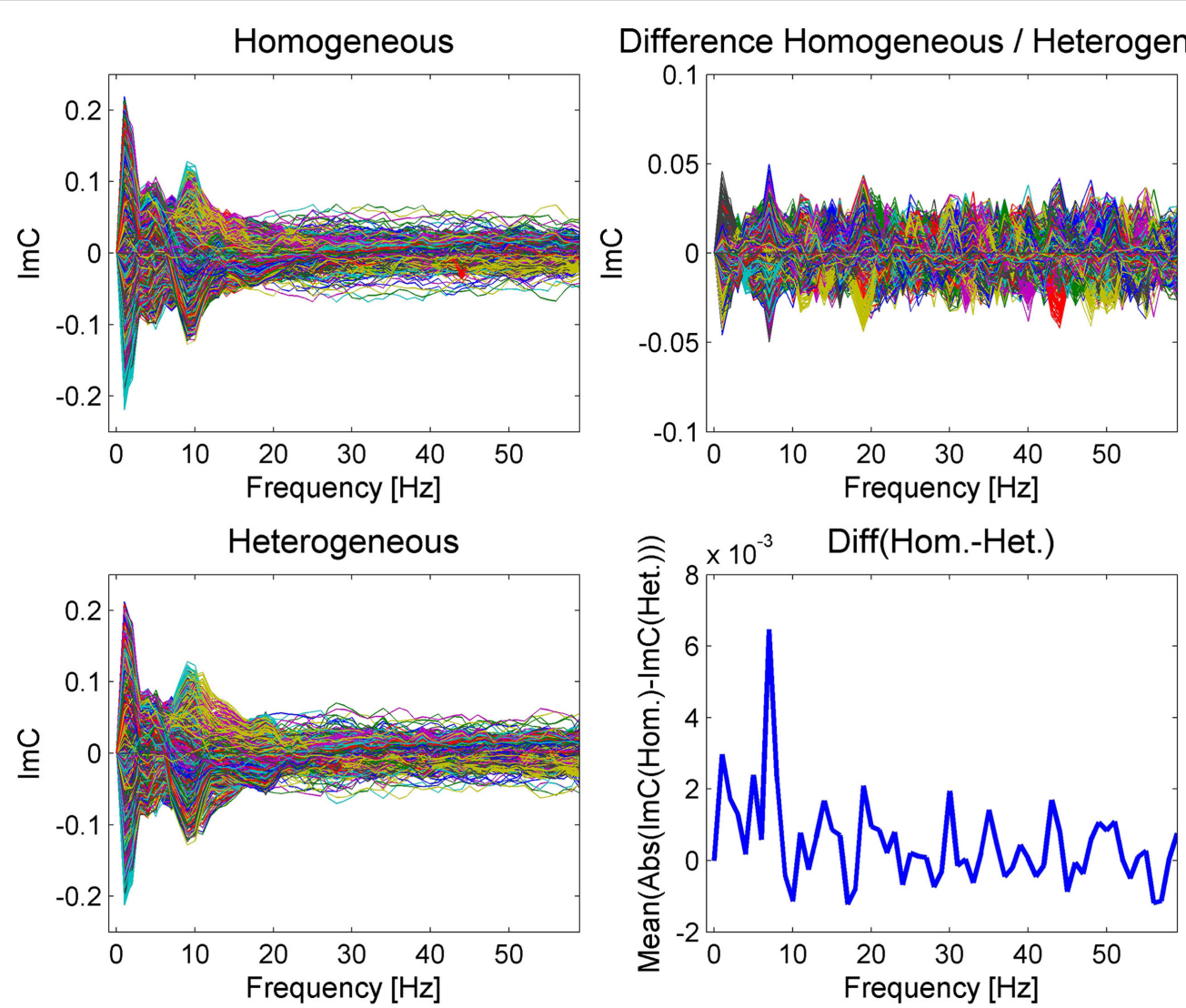

FIGURE 3 | The imaginary part of coherency is represented as a butterfly plot over frequency for the two different conditions on the left and for their difference on the upper right. The plot of the mean absolute value of the ImC over all channel differences (bottom right) reveals a prominent peak

at $7 \mathrm{~Hz}$, which is less visible in the individual condition plots due to overlapping modulations common to both conditions. Please note that the $\mathrm{ImC}$ is an antisymmetric measure meaning that the ImC of channel $A$ to channel $B$ equals minus the $I m C$ of channel $B$ to channel $A$.

in blue. In the lower plot the permutation test results for $7 \mathrm{~Hz}$ are displayed. One can see in both plots that the observed result at $7 \mathrm{~Hz}$ lies at the tail of the permutation distribution indicating that the measured effect unlikely occurred by chance. In fact, the $p$-value for $7 \mathrm{~Hz}$ was calculated to be $p=0.0029$ as only 5 out of 2000 permutation runs returned a higher result than the one observed. A Bonferroni correction for multiple comparisons at frequencies between 1 and $30 \mathrm{~Hz}$ yields a corrected alpha-level of $\alpha=0.05 / 30=0.0017$. Only correcting for a smaller range of frequencies, i.e., $1 \mathrm{~Hz} \leq \mathrm{f} \leq 15 \mathrm{~Hz}$, would lead to an alpha-level of $\alpha=0.0033$. In any case, the permutation test suggests the observed effect at $7 \mathrm{~Hz}$ to be reliable.

To further examine the ImC at $7 \mathrm{~Hz}$ spatially, we employ so called head-in-head plots to visualize bivariate interactions on channel level (Figure 5). The topographies at each electrode position represent the strength of connection (here the $\mathrm{ImC}$ ) between that given electrode and all other electrodes. For the interpretation of our data it is important to notice that as the ImC is antisymmetric, the connections shown are also antisymmetric, that is, inferences about the directionality of information flow cannot be made.

Despite the reduced effect size of the differences between the two conditions, statistical analyses yielded distinct coherency

pattern in theta oscillatory activity (Figure 5 bottom left). Genovese et al. (2002) motivate FDR correction with $q$-thresholds as high as 0.2 . We here employ statistical testing with an FDR correction procedure at a $q$-level of 0.1 (i.e., $90 \%$ of the detections are expected to be true detections) which confirmed significant differences between the blocking conditions. The uncorrected $p$-values at this level were below 0.00003 . We also note, that the difference in the coherency between the two experimental conditions (a value of imaginary coherency around \pm 0.05 ) is about two times smaller than the coherency for the single conditions. Differences between conditions are small compared to common effects. Furthermore, the statistical correction procedure is applied for many comparisons as we deal with interactions $(56 \times 56-56=3080)$. To summarize, we consider a classical Bonferroni correction as too conservative and an FDR $q$-level of 0.1 as appropriate.

As the obtained results on sensor level are not uniquely interpretable in terms of interacting brain sources, we estimated the underlying sources based on the imaginary part of the crossspectrum (see EEG Recording and Analysis). Figure 6 shows the results of the source localization as the two mainly interacting sources differing in the two experimental conditions. The source distributions are shown in four different views. Furthermore, the scalp topographies demixed by the MOCA algorithm 


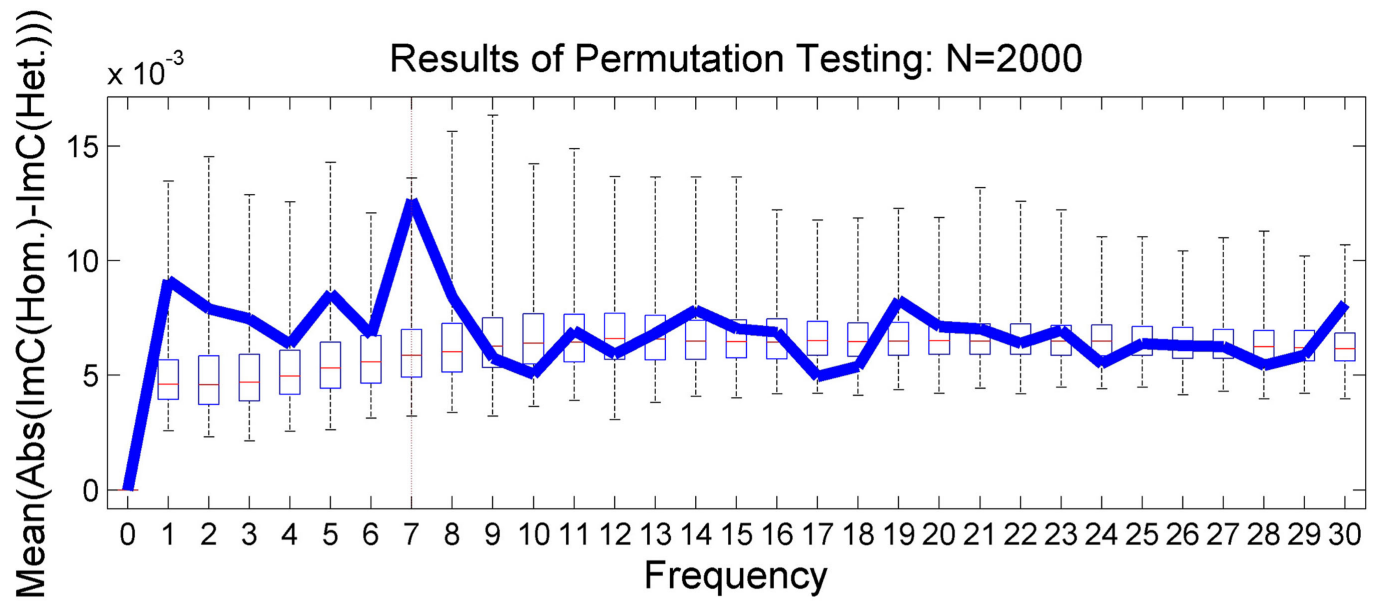

Permutation Histogram at $7 \mathrm{~Hz}$

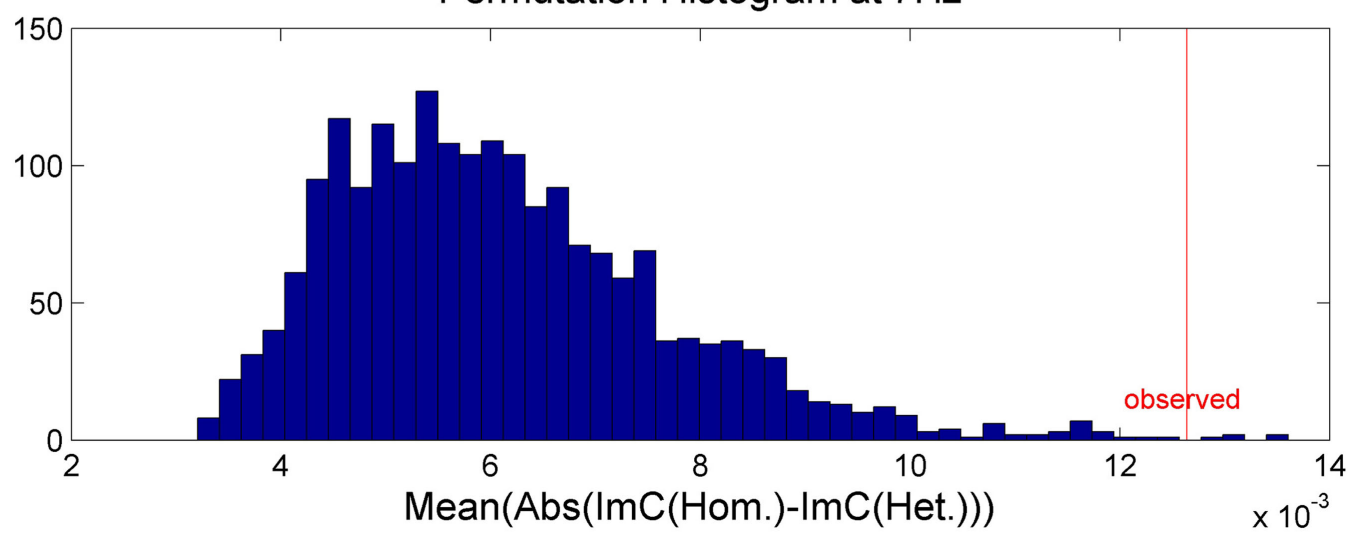

FIGURE 4 |The results of the permutation test. In the upper plot the results are presented for $1 \mathrm{~Hz} \leq f \leq 30 \mathrm{~Hz}$ as box plots showing the median and upper and lower quartiles. The whiskers indicate all permutation results outside of the quartiles. The results of the true class memberships are displayed in blue. The lower plot shows histogram of the permutation results for $7 \mathrm{~Hz}$ only. are illustrated (Marzetti et al., 2008). For the first source we mainly observe a fronto-central and an occipital activation. The second source shows predominantly left but also right-lateralized deep occipital activation and in addition right temporal activity. Although the inverse solution only gives a coarse picture of the involved brain regions it supports the statistically significant results obtained by the investigation of the ImC at sensor level.

To summarize, our findings provide evidence that semantic interference effects in language production are reflected in theta oscillation changes and in altering connectivity involving mainly frontal and occipital-temporal brain regions.

\section{DISCUSSION}

In the present study we investigated the communication of different large scale brain sites during an overt language production task with state of the art methods for the estimation of EEG functional connectivity. Up until now electrophysiological investigations of overt speech production, rare in general, are confined to ERP research (see, e.g., Indefrey, 2011 for a review). There is, to our knowledge only one (very recent) study that reports oscillatory activity during overt language production (Piai et al.,
2012), and not a single study on functional connectivity. Thus, the present study was designed to yield first evidence synchronization dynamics during overt speech production using a paradigm that taps into lexical-semantic processing components. We used a semantic blocking task in which objects were named in semantically homogeneous or heterogeneous blocks of trials. As shown before, a semantic interference effect was found with slower naming times in the homogeneous relative to the heterogeneous blocks. This effect is assumed to reflect the duration of lexical-semantic processing during speech planning.

We applied a measure for functional connectivity on sensor level, the ImC which is robust to artifacts of volume conduction in the sense that non-vanishing imaginary parts cannot be explained by independent sources. The converse, however, is not true. From a zero ImC one cannot explicitly conclude that no interacting sources are present. In fact, it is conceivable that true interactions occur at zero phase lag and are then missed by this measure. We here take a conservative viewpoint: since zero phase relations are ambiguous and can be explained equally by interacting and non-interacting sources we make no attempt to interpret the respective quantities in terms of brain connectivity. We also 


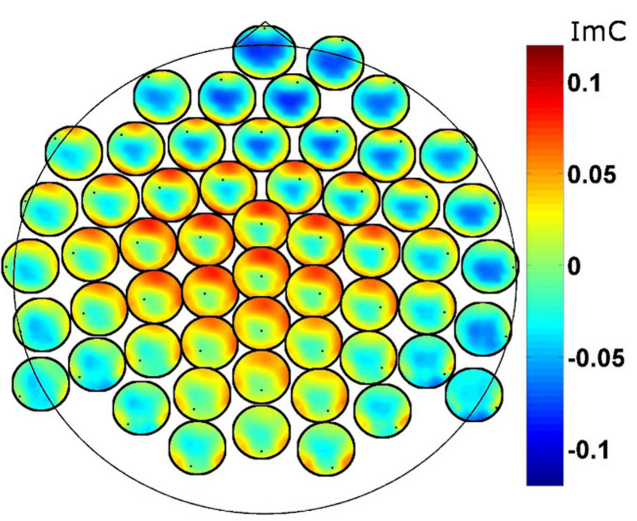

Homogeneous

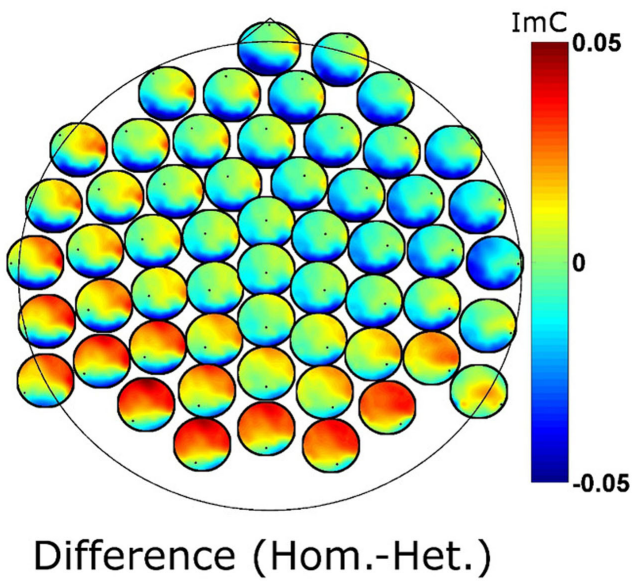

FIGURE 5 | Head-in-head plots for the imaginary part of coherency at $\mathbf{7 ~ H z}$ for the two conditions (top row) and for their difference (bottom left). The bottom right graph reports the FDR corrected $p$-values for the ImC

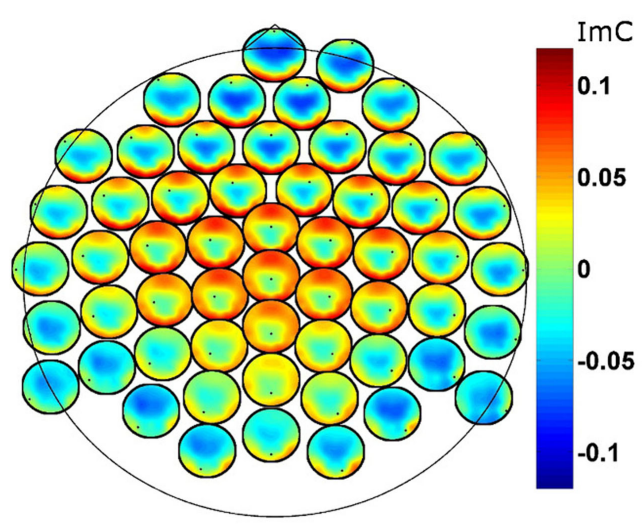

Heterogeneous

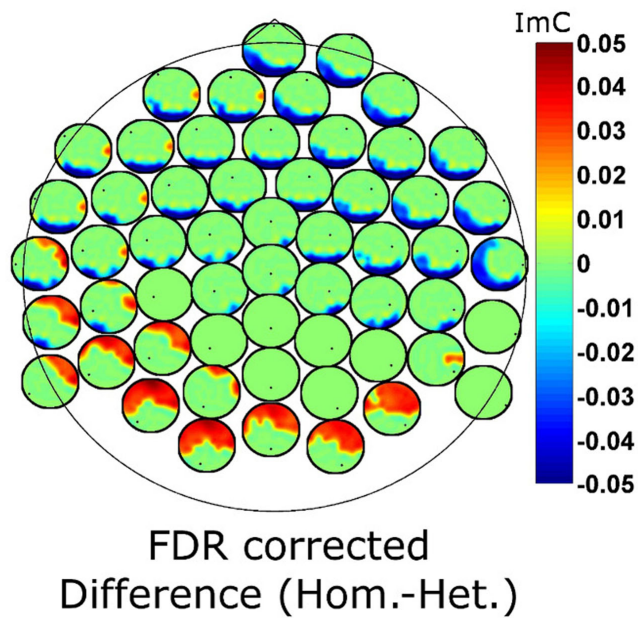

difference ( $p=0.1$ after correction). The tiny black dots represent the position of the reference electrode in terms of connectivity. Hot as well as cold colors code strong connectivity.

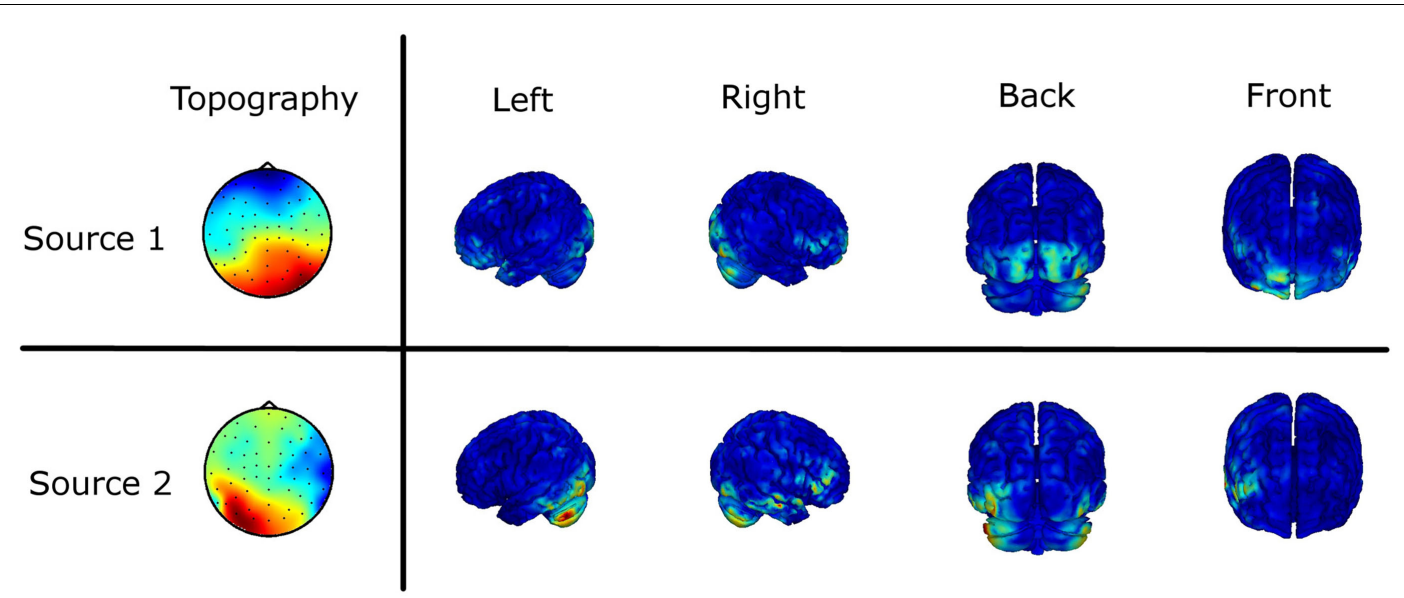

FIGURE 6 | Source localization of the main interaction of the difference in the two experimental conditions based on the imaginary part of the cross-spectrum. The two topographies (Topo View) are the eigenvectors of the subspace decomposition demixed by MOCA. The corresponding sources obtained by a weighted minimum norm estimate (WMNE) are shown in four different views. 
emphasize that by projecting the complex quantity coherency on the imaginary axis it becomes impossible to differentiate for a change of ImC between a change of the magnitude of coherency, i.e., the absolute value of coherency (=coherence), and a change of the phase relationship. Demanding to observe this difference and still retaining the robustness to volume conduction would require the application of non-linear methods, which is subject to ongoing research.

We here showed and explained a step by step scheme to determine necessary parameters and to estimate reliable macroscopic interaction of brain sources. We demonstrated the applicability of reliable connectivity measures such as the ImC (Nolte et al., 2004) as well as the estimation of the main interacting sources based on this measure.

To display the bivariate connections at a specific frequency on sensor level, we applied head-in-head plots. These plots provide a first view on the data in terms of connectivity and serves as a pre-processing before applying inverse calculations to obtain the underlying network of interacting sources. This has the advantage of diminishing artifacts and focusing on previously estimated robust and true interactions. The source estimation procedure we presented is based on subspace decomposition and, hence, focuses on the most prominent interacting brain regions differing in the experimental conditions.

The choice of a 1-s interval for our connectivity analyses (see above) may pose a potential problem. Because mean naming latencies are around $700 \mathrm{~ms}$, the interval includes not only planning stages but also articulatory processes which may contaminate the signal. However, we are confident that the length of the interval is not critical for two reasons. First, identical words were produced in the experimental conditions. Since the shape and topographical distribution of articulation-induced EEG artifacts are mainly determined by the phonetics of the utterance (e.g., Aristei et al., 2011; for a review, see Ganushchak et al., 2011), artifact-related activity should not contribute to condition-specific effects. Second, eye movement and muscle artifacts (that can be effectively removed with a 10 - to $12-\mathrm{Hz}$ low-pass filter) are typically reflected in higher frequency bands and should therefore not affect theta activity (e.g., Goncharova et al., 2003; Yuval-Greenberg et al., 2008). Thus, connectivity at theta frequencies is unlikely due to articulatory muscle artifacts. Overall, the ImC pattern was very similar in homogeneous and heterogeneous blocks, suggesting that these global interactions during word retrieval in the two blocking conditions involve overlapping functions and functional networks. Contrasting overt object naming in the homogeneous and heterogeneous condition we found small but reliable differences in interactions between left temporal and frontal areas and frontal and occipital areas in the theta band at $7 \mathrm{~Hz}$. Thus, differences in theta coherence are associated with semantic interference during language production. The associated frontal and temporal areas are known to play a role in lexical-semantic language processes (see below). Based on the general similarity of the ImC patterns, we speculate that the changes observed in the homogeneous condition may reflect quantitative differences in terms of task load or difficulty, rather than qualitative differences. Thus, we suggest that our data most likely reflect an enhanced effort of selecting a candidate from among competing alternatives in the mental lexicon. This selection process is known to be affected by semantic blocking, reflected in a semantic interference effect.

Our study presents, to our knowledge, the first report on theta oscillations and theta coherency changes in language production. However, our findings are in line with higher theta synchronizations at frontal-central and temporal regions reported in language comprehension tasks (c.f. Introduction). In comprehension, changes in theta oscillations and in theta coherency have been correlated with lexical-semantic retrieval. For instance, an increase in theta coherency at frontal electrodes was observed for semantic and syntactic violations in sentence processing (e.g., Bastiaansen et al., 2002). In line with assumptions that the language perception and production systems share lexical and semantic representations and, to some extent, processes in reversed order (Indefrey and Levelt, 2004; Indefrey, 2011), we suggest that the present findings reflect lexical-semantic processes during speech planning.

In line with this, our data seem compatible with recent fMRI and tDCS studies using the semantic blocking paradigm that have reported frontal (Wirth et al., 2011) and fronto-temporal activation patterns. The frontal activation has been taken to reflect selection from among competing alternatives stored in temporal regions (e.g., Schnur et al., 2005a,b, 2006). However, in these studies the frontal activation was found in the left inferior prefrontal cortex whereas in the present study we found connectivity patterns predominantly between right frontal and occipito-temporal sites. While a stronger involvement of left frontal sites would have been predicted, we can only speculate about the role of the right frontal regions in semantic blocking. Possibly, and converging with the hypothesis that repeated semantic blocking produces a refractory period of the activated items in semantic memory (e.g., Belke et al., 2005), and in line with studies showing right frontal activation for memory encoding of pictures (for a review, see Buckner et al., 1999), the right frontal distribution might reflect differences in the memory load between homogeneous an heterogeneous blocks, being higher for the homogeneous condition. This activation would be then transferred to the temporal regions where semantically related competitors become active. This would also be in line with previous studies showing a link between frontal theta oscillations and long term or working memory load (e.g., Klimesch et al., 2001; Bastiaansen et al., 2002).

Alternatively, theta oscillatory activity and theta coherency may reflect differences in long term or working memory (e.g., Klimesch et al., 2001; Bastiaansen et al., 2002; see Belke et al., 2005 for a compatible proposal based on behavioral interference effects in the semantic blocking paradigm). Furthermore, the data may be related to differential effects of top-down predictions induced by the semantic blocking contexts (e.g., Arnal et al., 2011). Future research should be performed to distinguish between functional networks related to more general processes and language - specific networks. Moreover, besides coherency analyses that convey information about "long range" interstructural connections, power spectral changes should be investigated in order to identify local neuronal oscillatory responses associated with lexical-semantic activation and selection during language production.

Interestingly, the estimation of source localization revealed an additional contribution of the cerebellum. Several prior studies have shown cerebellar activation and connectivity with frontal 
and prefrontal areas in different language production tasks (e.g., Desmond et al., 1998; Murdoch and Whelan, 2007). However, the precise role of the cerebellum in language production remains unclear. Thus, cerebellar activation has been associated with early production components such as semantic processing (e.g., Papthanassiou et al., 2000) and lexical retrieval (e.g., Marien et al., 2001) but also with late articulatory processes (e.g., Ackermann et al., 1998; see Murdoch, 2010 for a review). Cerebellar activation has also been reported in a recent study by Spalek and Thompson-Schill (2008) using the picture-word-interference paradigm (PWI) that is assumed to reflect similar semantic interference effects as the blocking paradigm. The authors interpreted the observed enhanced activation of the cerebellum by categorically related relative to unrelated word distractors in terms of lexical competition. Our results converge with these reports and may reflect lexical-semantic or articulatory aspects of language production.

\section{REFERENCES}

Abdel Rahman, R., and Aristei, S. (2010). Now you see it, and now again: semantic interference reflects lexical competition in speech production with and without articulation. Psychon. Bull. Rev. 17. 657-661.

Abdel Rahman, R., and Melinger, A. (2007). When bees hamper the production of honey: lexical interference from associates in speech production. J. Exp. Psychol. Learn. Mem. Cogn. 33, 604-614.

Abdel Rahman, R., and Sommer, W. (2003). Does phonological encoding in speech production always follow the retrieval of semantic knowledge? Electrophysiological evidence for parallel processing. Cogn. Brain Res. 16, 372-382.

Abdel Rahman, R., van Turennout, M., and Levelt, J. W. M. (2003). Phonological encoding is not contingent on semantic feature retrieval: an electrophysiological study on object naming. J. Exp. Psychol. Learn. Mem. Cogn. 29, 850-860.

Ackermann, H., Wildgruber, D., Daum, I., and Grodd, W. (1998). Does the cerebellum contribute to cognitive aspects of speech production? A functional magnetic resonance (fMRI) study in humans. Neurosci. Lett. 247, 187-190.

Aristei, S., Melinger, A., and Abdel Rahman, R. (2011). Electrophysiological chronometry of semantic context effects in language production. J. Cogn. Neurosci. 23, 1567-1586.

Arnal, L. H., Wyart, V., and Giraud, A. L. (2011). Transition in neural oscillations reflect prediction errors generated in audiovisual speech. Nat. Neurosci. 14, 797-801.
Baillet, S., Mosher, J. C., and Leahy, R. M. (2001). Electromagnetic brain mapping. IEEE Signal Process. Mag. 18, 14-30.

Bastiaansen, M. C. M., Oostenveld, R., Jensen, O., and Hagoort, P. (2008). I see what you mean: theta power increases are involved in the retrieval of lexical semantic information. Brain Lang. 106, 15-28.

Bastiaansen, M. C. M., Posthuma, D., Groot, P. F., and de Geus, E. J. (2002). Event-related alpha and theta responses in a visuo-spatial working memory task. Clin. Neurophysiol. 113, 1882-1893.

Bastiaansen, M. C. M., van der linden, M., Ter Keurs, M., Dijkstra, T., and Hagoort, P. (2005). Theta responses are involved in lexicosemantic retrieval during language processing. J. Cogn. Neurosci. 17, 530-541.

Belke, E., Meyer, A. S., and Damian, M. F. (2005). Refractory effects in picture naming as assessed in a semantic blocking paradigm. Q. J. Exp. Psychol. (Hove.) 58A, 667-692.

Bendat, J., and Piersol, A. (1971). Random Data; Analysis and Measurement Procedures. New York: WileyInterscience.

Benjamini, Y., and Hochberg, Y. (1995). Controlling the false discovery rate: a practical and powerful approach to multiple testing. J. R. Stat. Soc. Series B Stat. Methodol. 57, 89-300.

Brooker, B. H., and Donald, M. W. (1980). Contribution of speech musculature to apparent EEG asymmetries prior to vocalization. Brain Lang. 9, 226-245.

Brunner, D., Vasko, R., Detka, C., Monahan, J., Reynolds, C., and Kupfer, D. (1996). Muscle artifacts in the sleep EEG: automated detection and effect

To summarize, our results complement ERP evidence on lexical-semantic retrieval during language production. While ERPs provide precise temporal information about the different components of the speech production system, coherency analyses of neuronal oscillatory activity provide insight into functional connections within a broader time interval and at a specific frequency. Thus, coherency analyses complement the ERP data by shedding light on the "long distance" connections between large - scale neuronal assemblies involved in language production, namely, interactions between frontal and left occipito-temporal regions and the cerebellum. Thus, brain connectivity and event-related brain responses can provide an integrated picture on how language production is implemented in our brain.

\section{ACKNOWLEDGMENTS}

This work was supported by grants AB277 4 and 5 from the German Research Foundation (DFG) to Rasha Abdel Rahman.

on all-night EEG power spectra. J. Sleep Res. 5, 155-164.

Buckner, R. L., Kelley, W. M., and Petersen, S. E. (1999). Frontal cortex contributes to human memory formation. Nature 2, 311-314.

Christoffels, I. K., Firk, C., and Schiller, N. O. (2007). Bilingual language control: an event-related brain potential study. Brain Res. 1147, 192-208.

Costa, A., Strijkers, K., Martin, C., and Thierry, G. (2009). The time course of word retrieval revealed by eventrelated brain potentials during overt speech. Proc. Natl. Acad. Sci. U.S.A. 106, 21442-21446.

Damian, M. F., Vigliocco, G., and Levelt, W. J. M. (2001). Effects of semantic context in the naming of pictures and words. Cognition 81, B77-B86.

Desmond, J. E., Gabrieli, J. D. E., and Glover, G. H. (1998). Dissociation of frontal and cerebellar activity in a cognitive task: evidence for a distinction between selection and search. Neuroimage 7, 368-376.

Engel, A. K., Fries, P., and Singer, W. (2001). Dynamic predictions: oscillations and synchrony in top-down processing. Nat. Rev. Neurosci. 2, 704-716.

Eulitz, C., Hauk, O., and Cohen, R. (2000). Electroencephalographic activity over temporal brain areas during phonological encoding in picture naming. Clin. Neurophysiol. 111, 2088-2097.

Ewald, A., Marzetti, L., Zappasodi, F., Meinecke, F. C., and Nolte, G. (2011). Estimating true brain connectivity from EEG/MEG data invariant to linear and static transformations in sensor space. $\mathrm{Neu}$ roimage $60,476-488$.
Fries, P. (2005). A mechanism for cognitive dynamics: neuronal communication through neuronal coherence. Trends Cogn. Sci. (Regul. Ed.) 9, 474-480.

Ganushchak, L. Y., Christoffels, I. K., and Schiller, N. O. (2011) The use of electroencephalography in language production research: a review. Front. Psychol. 2:208. doi:10.3389/fpsyg.2011.00208

Ganushchak, L. Y., and Schiller, N. O. (2008). Motivation and semantic context affect brain error-monitoring activity: an event-related brain potentials study. Neuroimage 39, 395-405.

Ganushchak, L. Y., and Schiller, N. O. (2009). Speaking one's second language under time pressure: an ERP study on verbal self-monitoring in German-Dutch bilinguals. Psychophysiology 46, 410-419.

Genovese, C. R., Lazar, N. A., and Nichols, T. (2002). Thresholding of statistical maps in functional neuroimaging using the false discovery rate. Neuroimage 15, 870-878.

Goncharova, I. I., McFarland, D. J., Vaughan, T. M., and Wolpaw, J. R. (2003). EMG contamination of EEG: spectral and topographical characteristics. Clin. Neurophysiol. 114, 1580-1593.

Grözinger, B., Kornhuber, H. H., and Kriebel, J. (1975). Methodological problems in the investigation of cerebral potentials preceding speech: determining the onset and suppressing artifacts caused by speech. $\mathrm{Neu}$ ropsychologia 13, 263-270.

Hagoort, P., Hald, L., Bastiaansen, M., and Petersson, K. M. (2004). Integration of word meaning and world knowledge in language comprehension. Science 304, 438-441. 
Hauk, O. (2004). Keep it simple: a case for using classical minimum norm estimation in the analysis of EEG and MEG data, Neuroimage 21, 1612-1621.

Hirschfeld, G. H. F., Jansma, B., Bölte, J., and Zwitserlood, P. (2008). Interference and facilitation in overt speech production investigated with ERPs. Neuroreport 19, 1227-1230.

Huynh, H., and Feldt, L. S. (1976). Estimation of the box correction for degrees of freedom from sample data in the randomised block and splitplot design. J. Educ. Behav. Stat. 1, 69-82.

Indefrey, P. (2011). The spatial and temporal signatures of word production components: a critical update. Front. Psychol. 2:255. doi:10.3389/fpsyg.2011.00255

Indefrey, P., and Levelt, W. J. M. (2004). The spatial and temporal signatures of word production components. Cognition 92, 101-144.

Jescheniak, J. D., Schriefers, H., Garrett, M. F., and Friederici, A. D. (2002). Exploring the activation of semantic and phonological codes during speech planning with event-related brain potentials. J. Cogn. Neurosci. 14, 951-964.

Klimesch, W. (1999). EEG alpha and theta oscillations reflect cognitive and memory performance: a review and analysis. Brain Res. Rev. 29, 169-195.

Klimesch, W., Doppelmayr, M., Pachinger, T., and Russegger, $\mathrm{H}$. (1997). Event-related desynchronization in the alpha band and the processing of semantic information. Brain Res. Cogn. Brain Res. 6, 83-94.

Klimesch, W., Doppelmayr, M., Stadler, W., Pollhuber, D., Sauseng, P., and Rohm, D. (2001). Episodic retrieval is reflected by a processspecific increase in human electroencephalographic theta activity. Neurosci. Lett. 302, 49-52.

Krause, C. M., Gronholm, P., Leinonen, A., Laine, M., Sakkinen, A. L., and Soderholm, C. (2006). Modality matters: the effects of stimulus modality on the 4- to $30-\mathrm{Hz}$ brain electric oscillations during a lexical decision task. Brain Res. 1110, 182-192.

Kroll, J. F., and Stewart, E. (1994). Category interference in translation and picture naming: evidence for asymmetric connections between bilingual memory representations. J. Mem. Lang. 33, 149-174.

Lachaux, J. P., Rodriguez, E., Martinerie, J., and Varela, F. J. (1999). Measuring phase synchrony in brain signals. Hum. Brain Mapp. 8, 194-208.
Maess, B., Friederici, A. D., Damian, M., Meyer, A. S., and Levelt, W. J. M. (2002). Semantic category interference in overt picture naming: sharpening current density localization by PCA. J. Cogn. Neurosci. 14, 455-462. Marien, P., Engelborghs, S., Fabbro, F. and De Deyn, P. P. (2001). The lateralized linguistic cerebellum: a review and a new hypothesis. Brain Lang. 79, 580-600.

Marzetti, L., Del Gratta, C., and Nolte, G. (2008). Understanding brain connectivity from EEG data by identifying systems composed of interacting sources. Neuroimage 42, 87-98.

Moore, D. S., and McCabe, G. P. (2003). Introduction to the Practice of Statistics, 5th Edn. New York: W. H. Freeman.

Murdoch, B. E. (2010). The cerebellum and language: historical perspective and review. Cortex 46, 858-868.

Murdoch, B. E., and Whelan, B. M. (2007). Language disorders subsequent to left cerebellar lesions: a case for bilateral cerebellar involvement in language? Folia Phoniatr. Logop. 59, 184-189.

Nolte, G., Bai, O., Wheaton, L., Mari, Z., Vorbach, S., and Hallett, M. (2004). Identifying true brain interaction from EEG data using the imaginary part of coherency. Clin. Neurophysiol. 115, 2292-2307.

Nolte, G., Meinecke, F. C., Ziehe, A., and Mueller, K. R. (2006). Identifying interactions in mixed and noisy complex systems. Phys. Rev. E Stat. Nonlin. Soft Matter Phys. 73, 051913.

Nunez, P. L., Silberstein, R. B., Shi, Z., Carpenter, M. R, Srinivasan, R., Tucker, D. M., Doran, S. M., Cadusch, P. J., and Wijesinghe, R. S. (1999) EEG coherency II: experimental comparisons of multiple measures. Clin. Neurophysiol. 110, 469-486.

Papthanassiou, D., Etard, O., Mellet, E., Zago, L., Mazoyer, B., and TzourioMazoyer, W. (2000). A common language network for comprehension and production: a contribution to the definition of language epicenters with PET. Neuroimage 11, 347-357.

Piai, V., Roelofs, A., and van der meij, R. (2012). Event-related potentials and oscillatory brain responses (associated) with semantic and Stroop-like interference effects in overt naming. Brain Res. 1450, 87-101.

Plonsey, R., and Heppner, D. (1967). Considerations of quasi-stationarity in electrophysiological systems. Bull. Math. Biophys. 29, 657-664.

Rohm, D., Klimesch, W., Haider, H., and Doppelmayr, M. (2001). The role of theta and alpha oscillations for language comprehension in the human electroencephalogram. Neurosci. Lett. 310, 137-140.

Schmitt, B. M., Münte, T. F., and Kutas, M. (2000). Electrophysiological estimates of the time course of semantic and phonological encoding during implicit picture naming. Psychophysiology 37, 473-484.

Schmitt, B. M., Schiltz, K., Zaake, W. Kutas, M., and Münte, T. F. (2001). An electrophysiological analysis of the time course of conceptual and syntactic encoding during tacit picture naming. J. Cogn. Neurosci. 13, 510-522.

Schnur, T. T., Hirshorn, E., and Thompson-Schill, S. L. (2005a). "Mapping semantic interference during picture naming: an fMRI study," in Paper presented at the Cognitive Neuroscience Society, New York.

Schnur, T. T., Lee, E., Coslett, H. B. Schwartz, M. F., and ThompsonSchill, S. L. (2005b). When lexical selection gets tough, the LIFG gets going: a lesion analysis study of interference during word production. Brain Lang. 95, 12-13.

Schnur, T. T., Schwartz, M. F., Brecher, A., and Hodgson, C. (2006). Semantic interference during blockedcyclic naming: evidence from aphasia. J. Mem. Lang. 54, 199-227.

Schoeffelen, J., and Gross, J. (2009). Source connectivity analysis with MEG and EEG. Hum. Brain Mapp. 30, 1857-1865.

Singer, W. (1999). Neuronal synchrony: a versatile code for the definition of relations Neuron 24, 49-65.

Spalek, K., and Thompson-Schill, S. L. (2008). Task-dependent semantic interference in language production: an fMRI study. Brain Lang. 107, 220-228.

Stinstra, J. G., and Peters, M. J. (1998). The volume conductor may act as a temporal filter on the ECG and EEG. Med. Biol. Eng. Comput. 36, 711-716.

Storey, J. D. (2002). A direct approach to false discovery rates. J. R. Stat. Soc. Series B Stat. Methodol. 64, 479-498.

Strijkers, K., and Costa, A. (2011). Riding the lexical speedway: a critical review on the time course of lexical access in speech production. Front. Psychol. 2:356. doi:10.3389/fpsyg.2011.00356

Strijkers, K., Costa, A., and Thierry, G. (2010). Tracking lexical access in speech production: electrophysiological correlates of word frequency and cognate effects. Cereb. Cortex 20 912-928. van Turennout, M., Hagoort, P., and Brown, C. M. (1997). Electrophysiological evidence on the time course of semantic and phonological processes in speech production. J. Exp. Psychol. Learn. Mem. Cogn. 23, 787-806.

van Turennout, M., Hagoort, P., and Brown, C. M. (1998). Brain activity during speaking: from syntax to phonology in $40 \mathrm{msec}$. Science 280, 572-574.

Varela, F., Lachaux, J. P., Rodriguez, E., and Martinerie, J. (2001). The brainweb: phase synchronization and large-scale integration. Nat. Rev. Neurosci. 4, 229-239.

Wirth, M., Abdel Rahman, A., Kuenecke, J., Koenig, T., Horn, H., Sommer, W., and Dierks, T. (2011). Effects of transcranial direct current stimulation (tDCS) on behaviour and electrophysiology of language production. Neuropsychologia 49, 3989-3998.

Woestenburg, J. C., Verbaten, M. N., and Slangen, J. L. (1983). The removal of the eye-movement artifact from the EEG by regression analysis in the frequency domain. Biol. Psychol. 16, 127-114.

Wohlert, A. B. (1993). Event-related brain potentials preceding speech and nonspeech oral movements of varying complexity. J. Speech Hear. Res. 36, 897-905.

Yuval-Greenberg, S., Tomer, O., Keren, A. S., Nelken, I., and Deouell, L. Y. (2008). Transient induced gammaband response in EEG as a manifestation of miniature saccades. Neuron 58, 429-441.

Conflict of Interest Statement: The authors declare that the research was conducted in the absence of any commercial or financial relationships that could be construed as a potential conflict of interest.

Received: 23 December 2011; accepted: 10 May 2012; published online: 07 June 2012.

Citation: Ewald A, Aristei S, Nolte G and Rahman RA (2012) Brain oscillations and functional connectivity during overt language production. Front. Psychology 3:166. doi: 10.3389/fpsyg.2012.00166 This article was submitted to Frontiers in Language Sciences, a specialty of Frontiers in Psychology.

Copyright (C) 2012 Ewald, Aristei, Nolte and Rahman. This is an open-access article distributed under the terms of the Creative Commons Attribution Non Commercial License, which permits noncommercial use, distribution, and reproduction in other forums, provided the original authors and source are credited. 